\title{
Vitamin D-A Possible Natural Origin Agent for Medical Treatment of Uterine Leiomyomas in View of Safety, Cost Effectiveness Over SPRM's-A Short Communication
}

ISSN: 2637-8078

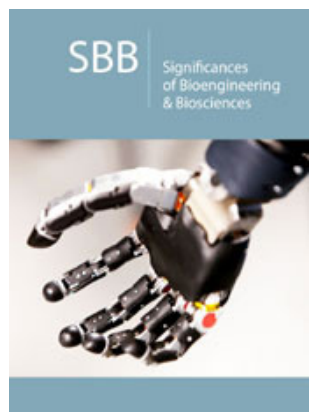

*Corresponding author: Kulvinder Kochar K, Centre for Human reproduction, India

Submission: 眥June 14, 2019

Published: 酹July 12, 2019

Volume 3 - Issue 4

How to cite this article: Kulvinder Kochar K, Gautam A, Mandeep S. Vitamin D-A Possible Natural Origin Agent for Medical Treatment of Uterine Leiomyomas in View of Safety, Cost Effectiveness Over SPRM's-A Short Communication. Significances Bioeng Biosci.3(4). SBB.000566.2019.

DOI: 10.31031/SBB.2019.03.000566

Copyright@ Kulvinder Kochar K, This article is distributed under the terms of the Creative Commons Attribution 4.0 International License, which permits unrestricted use and redistribution provided that the original author and source are credited.

\author{
Kulvinder Kochar $\mathrm{K}^{1 *}$, Gautam Allahbadia ${ }^{1}$ and Mandeep Singh ${ }^{2}$ \\ ${ }^{1}$ Centre for Human reproduction, India \\ ${ }^{2}$ Consultant Neurologist, India
}

\section{Introduction}

Human uterine leiomyomas (ULs) being the commonest neoplasms affecting women of reproductive age affect African American (AA) much more as compared to Caucasian (CC) women. One of the possible reasons thought is in view of higher incidence of hypovitaminosis $\mathrm{D}$ in this group. We have been trying to look for a permanent medical agent that can be used long-term for fibroid treatment instead of the earlier selective progesterone receptor modulators(SPRM)'s of which ulipristal acetate has been recommended by the FDA but in view of its side effects like progesterone associated endometrial changes(PAECs), it can't be used for long-term. Thus, in an endeavor to find a medical therapy that can be used for long term without any adverse side effects Vitamin D is getting pursued with its effect on Wnt/betacatenin pathway by which it can affect the MED12 status, a possible cause of etiopathogenesis of UL. Human uterine leiomyomas (ULs) affect roughly 70\% of women of reproductive age [1], being the most common benign estrogen-dependent tumors that develop during the reproductive period [2,3]. It is most prevalent in African American (AA) women (73\%), in comparison with Caucasian (CC) women (45\%) [4]. Though they are benign, nonetheless these tumors cause significant morbidity, being the main cause for hysterectomy and also for both gynecologic dysfunctions associated with reproductive dysfunction that varies from heavy menses along with pelvic pain to infertility, recurrent miscarriage and preterm labour. The etiopathogenesis of ULs is mostly not clear, yet MED12 mutations are the most common driving mutations accounting for roughly $70 \%$ of UL's $[5,6]$.

The canonical Wnt/ $\beta$-catenin pathway is a well characterized cell signaling pathway, connected to the formation of many kinds of tumour. The activation occurs downstream of the interaction of a Wnt ligand with the Frizzle/LRP co-receptor complex, that prevents the degradation of $\beta$-catenin targeted genes in human myometrial cells [7]. Earlier Al-Hendy et al. [7] showed that $\beta$-catenin signaling was activated in the myometrium of UL's with MED12 mutation in comparison of the normal adjacent myometrium. The MED $12131 \mathrm{G}>\mathrm{A}$ mutations cause a raised expression of $\beta$-catenin targeted genes in human myometrium cells [7]. A paracrine role for Wnt/ $\beta$-catenin signaling has been proposed by Ono et al. [8] that helps differentiated myometrial cells or UL cells to send mitogenic signals to the nearby myometrial stem cells in response to estrogen (E)and progesterone(P), that causes growth of ULs. A woman has a threefold greater incidence rate and relative risk of ULs than Caucasians (CC)women. Basis of this risk differentiation is not clear, some current studies point to hypovitaminosis D as the main culprit, since AA women have a ten-times >risk of Vitamin deficiency as compared with CC women [5]. With these findings with the more recent observations that vitamin D3 and its potent nonhypercalcemic analog paricalcitol effectively depressed UL growth in animal models [5], implicates that analogs of Vitamin D and Vitamin D might be promising to prevent or for early therapy. Further Al-Handy et al. [7] demonstrated that silencing of Vitamin D receptor (VDR) in normal myometrial cells induces expression of Wnt $4 / \beta$-catenin and stimulates a fibrotic process, which involves raised cell proliferation along with extracellular matrix production [9]. 
Recently Corachan et al. [10] evaluated the differential expression of proliferation and apoptosis -related markers like PCNA, BCL2 and BAX) along with WISP1(Wnt inducible signaling pathway protein) in various matched myometrial and UL tissues. Initially they showed that the upregulation of PCNA and WISP1 was seen in ULs that is similar to the previous observations. But they did not find impairment in apoptosis in ULs they saw, probably patient variabilities might be a contributing factor. Nevertheless, on evaluation of these markers in matched patient tissues is definitely more reliably. They then examined the effect of Vitamin D on induction of isolated primary cell growth arrest along with decrease in PCNA protein expression. With the use of primary cells to measure Vitamin D effect is a point that adds strength in contrast to immortalized cell lines. Furthermore, Wnt signaling along with apoptosis specific polymerase chain reaction arrays have been utilized for determining the Vitamin D response on gene expression which are involved in crucial pathways. More in depth studies might be carried out from observing these studies in future.

Though the efficiency of Vitamin D therapy is correlated with patient disparity along with levels of VDR, the ethnicity of the collected patient samples was not described in this article. If the observations related to the levels of VDR in ULs are present in this study one will obtain more detailed information regarding the efficiency of treatment with vitamin D3 in ULs. Since this correlation has been ignored, this relation might explain why apoptotic effect of Vitamin D treatment on isolated primary cells in this study is not consistent with the earlier findings that showed that Vitamin D therapy causes apoptosis in UL cells [5], since VDR is a nuclear receptor which responds to its ligand (i.e. Vitamin D), as demonstrated earlier [5,9]. Hence need for dividing subgroup into the samples tested as per VDR expression might demonstrate marked effect following Vitamin D therapy. Likewise testing the MED12 mutation status in the tissues might give helpful knowledge for understanding the Wnt polymerase chain reaction arrays data once Vitamin D therapy has been given, in view ofMED12 mutation has been demonstrated to modulate Wnt/ $\beta$-catenin signaling. Hence pooling all the samples may dilute any effect and lead to minimal changes of gene expression secondary to Vitamin D therapy. Further measuring the protein levels of particular markers might have greater impact. Nuclear translocation of $\beta$-catenin instead of expression levels have been demonstrated to be responsible for etiopathogenesis of particular cancers. Hence measurement of expression of nuclear $\beta$-catenin expression in UL's might indicate the activation of this particular pathway more adequately. Further changes in $\beta$-catenin's expression i.e., decreased levels after testing efficacy of treatments like Vitamin D as anti-UL therapy of the modalities available. Moreover, since UL's are derived from monoclonal stem cells, genome wide analysis of Vitamin D's effect is needed. Earlier we reviewed the different approaches for medical management of fibroids [11], instead of the surgical or radiologic management which are not desired by the patient specially if she wants preservation of fertility in future. Hormonal therapies are designed to blunt the growth stimulatory actions of $\mathrm{E}$ and P on UL growth like selective progesterone receptor modulators (SPRM) which are approved for a short time in view of complications like progesterone associated endometrial changes (PAEC) on long term use. Ciebiera et al. [12] reviewed how Vitamin D might be obtained and its metabolic pathways (Figure 1). Further they showed the influence of Vitamin D on UL (Figure 2). Further they compared effects of Vitamin D, Gn RH analogs and SPRM'S (Figure 3). As per Ali et al there is a potential synergism between Vitamin D and ulipristal acetate [13,14]. Further Vitamin D also has an effect on extracellular matrix implicated in UL pathogenesis [15]. Thus, for long term therapies that are targeted for effective UL size decrease and ameliorate the common symptoms are actively needed for addressing this. These would not be affecting future fertility and would be safe and cost effective in view of natural origin of Vitamin $\mathrm{D}$ and its availability, hence it might appear the safest, cheaper option.

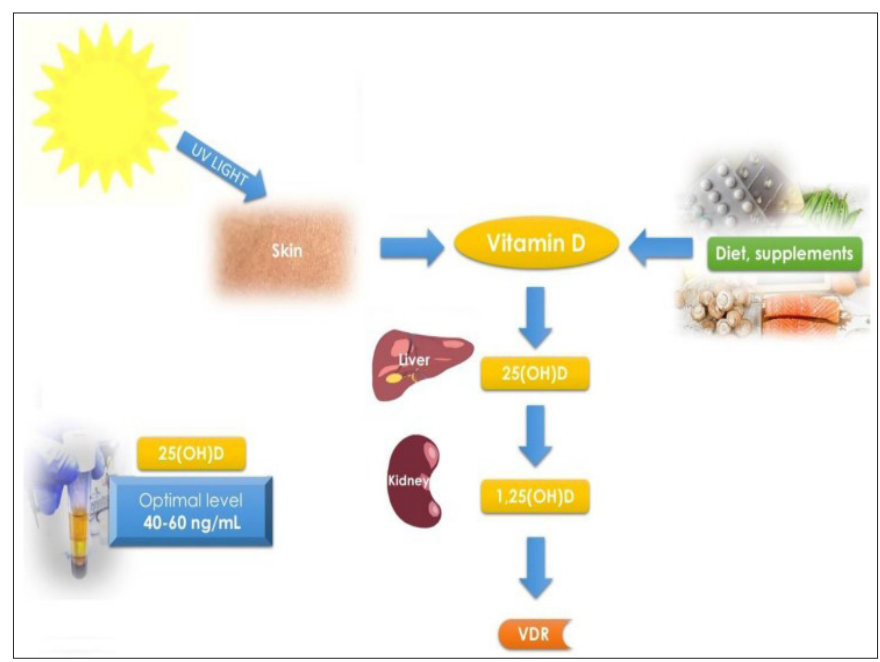

Figure 1: Courtesy [12]. Vitamin D metabolism and schematic pathways. Diet, supplements and sunlight are the major source of vitamin D in humans. Vitamin D is synthetized in skin from 7-ehydrocholesterol. In further steps liver converts it to $25(\mathrm{OH}) \mathrm{D}$ and then kidney to $1,25(\mathrm{OH}) \mathrm{D}$. Optimal vitamin D serum levels were described as $25(\mathrm{OH}) \mathrm{D}$ of $40-60 \mathrm{ng} / \mathrm{mL}$. 


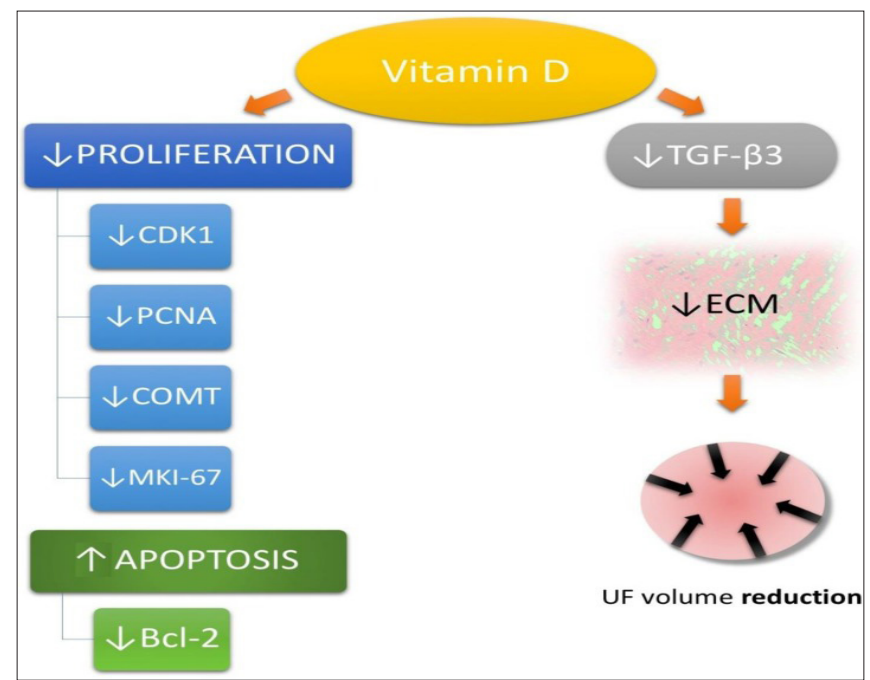

Figure 2: Curtesy [12]. Vitamin D influence on uterine fibroid (UF) pathophysiological pathways. Cyclindependent kinase 1 (CDK1), proliferating cell nuclear antigen (PCNA), catechol-O methyltransferase (COMT), Bcl2 protein, proliferation marker protein Ki-67 (MKI-67), extracellular matrix (ECM), transforming growth factor beta 3 (TGF- $\beta 3$ ), uterine fibroid (UF).

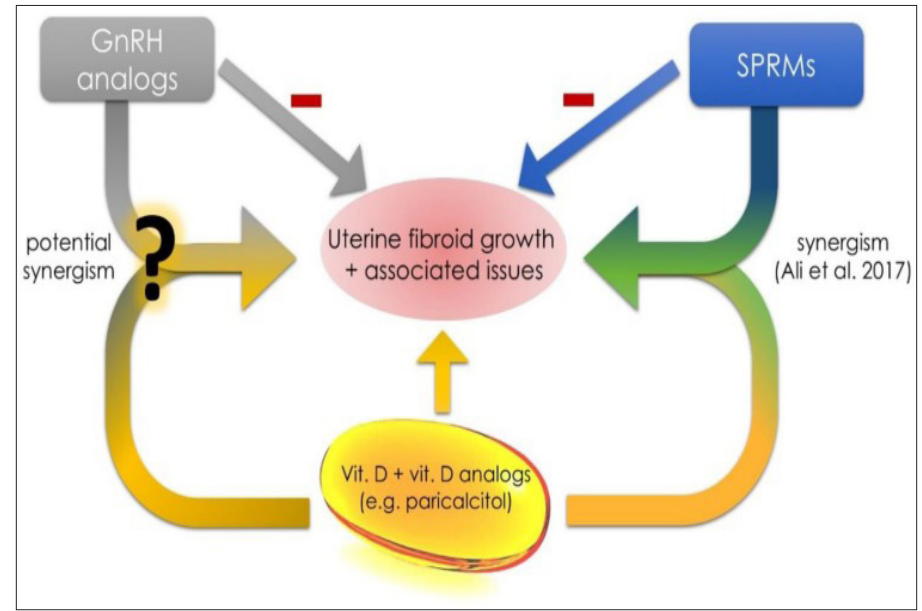

Figure 3: Courtesy [12]. Vitamin D, GnRH analogs and SPRMs. According to Ali et al. there is a potential synergism between vitamin $\mathrm{D}$ and ulipristal acetate [140]. New data about between vitamin D, vitamin $\mathrm{D}$ analogs and other mostly used drugs in UF therapy is necessary to find other synergisms. Gonadotropin releasing hormone (GnRH), selective progesterone receptor modulator (SPRM).

\section{Conclusion}

UL's being one of the commonest neoplasms in women of reproductive age require treatment. Earlier TAH was considered the only treatment or operative myomectomy/laparoscopic one was the only answer. After decade of trying for development of medical management still we have not managed to develop an effective treatment with SPRM, the commonest used of which ulipristal acetate has been approved but still flaw is it can't be used for long term in view of side effects like PAEC. One of the major proposed causes of development of UL's is MED12 mutations and Vitamin $D$ has been shown to act via Wnt/ $\beta$-catenin pathway to affect these uterine leiomyomas. Further studies are going on to ensure that this is the permanent medical treatment for UL's we have been looking for.

\section{References}

1. Practice guidelines of the American society for reproductive medicine (2017) Removal of myomas in asymptomatic patients to improve fertility and/or reduce miscarriage rate: A guideline. Fertil Steril 108(3): 416-425.

2. Cramer SF, Patel A (1990) The frequency of uterine leiomyomas. Am J Clin Pathol 94(4): 435-438.

3. Buttram VC, Reiter RC (1981) Uterine leiomyomas: Etiology, symptomatology and management. Fertil Steril 36(4): 433-445.

4. Baird DD, Dunson DB, Hill MC, Cousins D, Schectman JM (2003) High cumulative incidence of uterine leiomyomas in black and white women: ultrasound evidence. Am J Obstet Gynecol 188(1): 100-107. 
5. Brakta S, Diamond JS, Al-Hendy A, Diamond MP, Ismail N, et al. (2015) Role of Vitamin D in uterine fibroid biology. Fertil Steril 104(3): 698-706.

6. Kulvinder KK, Allahbadia GN, Singh M (2019) Moving towards developing stable medical treatments for uterine leiomyomas that can be used for long on the bases of molecular mechanisms like MED12 mutation status. EC Gynecology 8(6): 1-3.

7. Al-Hendy A, Laknaur A, Diamond MP, Ismail N, Boyer TG, et al. (2017) Silencing Med 12 gene reduces proliferation of human leiomyoma cells mediated via Wnt/beta-catenin signaling pathway. Endocrinology 158(3): 592-603.

8. Ono M, Yin P, Navarro A, Moravek MB, Coon JS, et al. (2013) Paracrine actions of WNT/beta-catenin pathway in uterine leiomyoma stem cells promote tumor growth. Proc Natl Aca Sci USA 110(42): 17053-17058.

9. Al-Hendy A, Diamond MP, Boyer TG, Halder SK (2016) Vitamin D3 inhibits Wnt/beta-catenin and MTOR signaling pathway $s$ in human uterine fibroid cells. J Clin Endocrinol Metab 101(4): 1542-1551.

10. Corachan A, Ferrero H, Aquilar A, Garcia N, Monleon J, et al. (2019) Inhibition of tumor cell proliferation inhuman uterine leiomyomas by vitamin D via Wnt/beta-catenin pathway. Fertil Steril 111(2): 397-407.
11. Kulvinder KK, Allahbadia GN, Singh M (2015) Medical management of leiomyomas: emphasis for different geographical regions. J Gynecol Neonatal Biol 1(2): 1-8.

12. Ciebiera M, Wlodarczyk M, Ciebiera M, Zareba K, Lukaszuk K, et al. (2018) Vitamin D and uterine fibroids-review of the literature and Novel Concepts. International J Molecular Sciences 19(7): 2051.

13. Ali MLA, Shaheen S, Sabri M, Al-Hendy A (2017) Vitamin D synergizes the antiproliferative, apoptotic, antifibrotic and anti-inflammatory effects of ulipristal acetate against human uterine fibroids. Fertil Steril 2017: 108(3): e66.

14. Ciebiera M, Meczekalski B, Lukaszuk K, Jakiel G (2019) Potential synergism between ulipristal acetate and Vitamin $\mathrm{D}_{3}$ in uterine fibroid pharmacotherapy-2 case studies. Gynaecol Endocrinol 35(6): 473-437.

15. Islam MS, Ciavattini A, Petraglia F, Castellucci M, Ciarmela P (2018) Extracellular matrix in Uterine leiomyoma pathogenesis: a potential target for future therapeutics. Hum Reprod Update 24(1): 59-85. 